Results from ROSAT \& GRO

\& other recent high energy astrophysics missions

Chairman \& Editor: J. Trümper 


\title{
RECENT GINGA RESULTS ON EXTRAGALACTIC X-RAY SOURCES
}

\author{
Y. TANAKA \\ Institute of Space and Astronautical Science \\ 3-1-1 Yoshinodai, Sagamihara, Kanagawa-ken 229, Japan
}

ABSTRACT. Many active galactic nuclei (AGNs) have been observed from Ginga, and their spectra as well as the time variations have been studied. Study of the sky brightness fluctuation has given a new result related to the cosmic $X$-ray background.

\section{Outline of the Ginga satellite}

The X-ray astronomy observatory Ginga, launched in February 1987 , carries a set of proportional counters of a total $4000 \mathrm{~cm}^{2} \mathrm{X}$-ray collecting area (LAC), together with an all sky monitor (ASM) and a gamma-ray burst detector (GBD). Ginga continues to perform observations normally, but is expected to end its orbital life before the end of 1991 .

\section{Energy spectra of AGNS}

A number of AGNs have been observed and their energy spectra, including those of $\sim 20$ QSOs, have been determined. Figure 1 shows the distribution of the power-law slope in the range $2-20 \mathrm{keV}$ for various classes of AGNs. The average slope (energy index) for Sefert 1 galaxies and QSOS is found to be canonical value; 0.7 , while the slopes for BL Lac objects are systematically steeper and more scattered.

Thanks to high statistical accuracy obtained from Ginga, a significant spectral features around $6-7 \mathrm{keV}$ have been detected. The observed spectra of Seyfert 1 galaxies can be well explained as comprising a power-law component (direct beam from the central source) and a reflected component from surrounding cool matter (probably the accretion disk) through multiple Compton scattering, accompanied by a fluorescent iron emission line (Pounds et al. 1990; Matsuoka et al. 1990).

The observed spectra of Seyfert 2 galaxies can be interpreted to be the case in which the central power-law source is heavily obscured by a surrounding matter. Otherwise, Sefert 1 and 2 galaxies are considered to be the same, but simply viewed at different inclination angles (Awaki et al. 1990; Awaki 1991). 


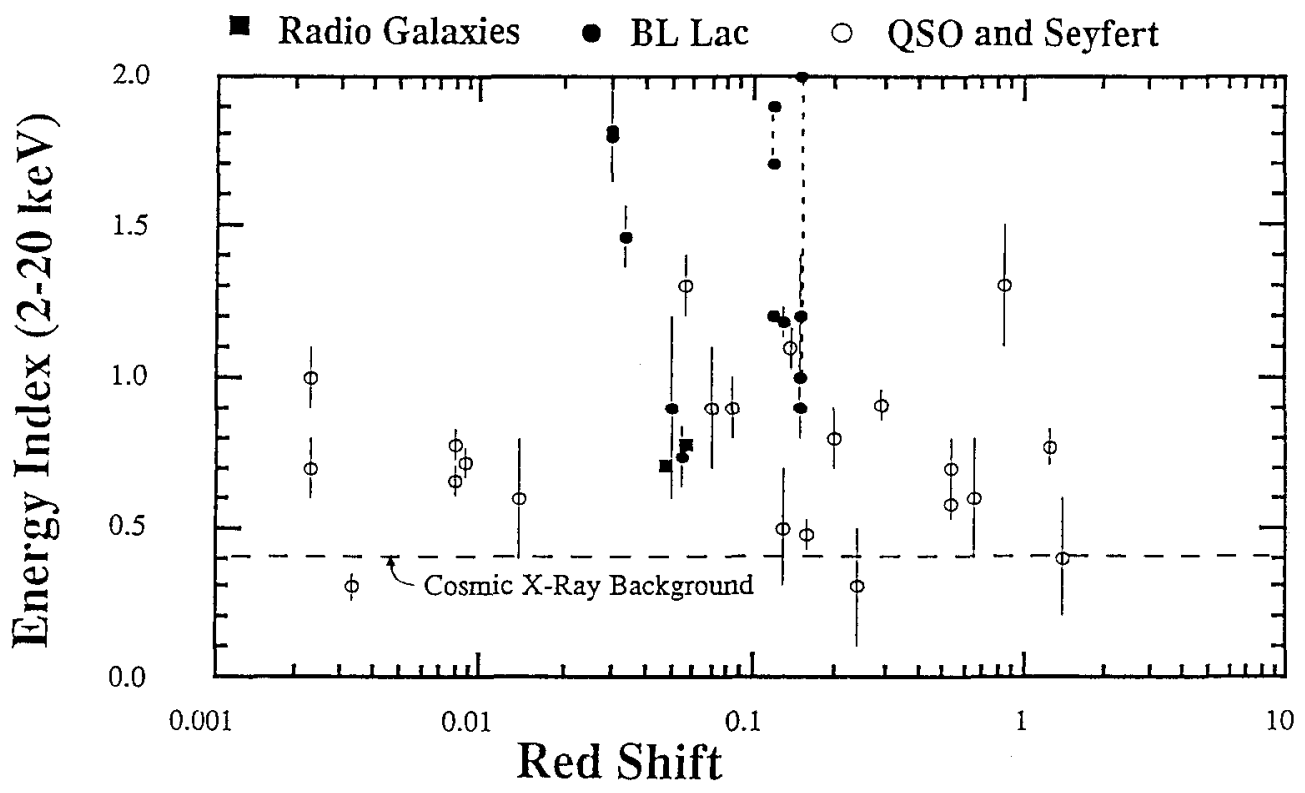

Fig. 1. Energy index distribution of AGNs observed from Ginga.

3. Similarities between AGNs and the galactic black-hole candidates

We note close similarities in the observed $X$-ray characteristics between AGNs and the galactic black hole candidates (see Paper by Y. Tanaka in this Joint Discussion: "Recent Ginga Results on Galactic X-Ray Binaries"). Chaotic intensity variation of the power-law component on time scales down close to the dynamic time scale. The power-law index distributed in the range, $0.4-1.5$, with the average value $\sim 0.7$. For each individual source, the power-law index remains constant against intensity changes of all time scales. Power-law spectrum extends beyond $100 \mathrm{keV}$ or higher. These facts suggest that, despite many orders of magnitude difference in the black hole mass and the system scale, the mechanism of generating the power-law radiation is common to both classes of sources, and that the site of the emission is located very near the central black hole.

In addition, the spectral features seen in Seyfert 1 galaxies around 6-7 keV are also present in the galactic black hole candidates with a power-law spectrum such as Cyg X-1 (Tanaka 1990), suggesting a similar accretion disk structure if scaled to the black hole mass.

\section{Rapid luminosity changes of QSOS}

Rapid luminosity variations have been observed from many Seyfert galaxies and BL Lac objects. Of particular interest are the cases of rapid changes observed from two high-luminosity QSOS. A luminosity 
increase of $20 \%$ in less than $45 \mathrm{~min}$. was observed from $3 \mathrm{C} 279$ (Makino et al. 1989). A more dramatic case was observed from PKS 0558-504 in which luminosity increased by $70 \%$ within $3 \mathrm{~min}$. and returned to the previous level after $20 \mathrm{~min}$. (Remillard et al. 1991). In both cases, the rate of increase $(\mathrm{dL} / \mathrm{dt})$ is an order of magnitude larger than the theoretical limit which holds for the case of spherical accretion (Cavallo and Rees 1978; Fabian 1979): the apparent energy conversion efficiency exceeds $100 \%$, whereas the actual efficiency can hardly be greater than $10 \%$. The authors interpret these rapid rises to be the case of relativistic beaming.

In this regard, it is important to note that the power-law index did not change through these rapid increases. This fact requires that the region responsible for the "flare" enits X-rays of the same spectral form as that of the persistent emission, while moving at a relativistic speed.

\section{Cosmic X-ray background}

The origin of an intense cosmic X-ray background (CXB) has been a longstanding important question. A substantial fraction of $\mathrm{CXB}$ has been resolved into discrete sources with the Einstein observatory and more recently by ROSAT. However, CXB over a wider range above $2 \mathrm{keV}$ which contains most part of energy of $\mathrm{CXB}$ is yet to be resolved.

Sky brightness fluctuations have been studied extensively from Ginga in the range $2-10 \mathrm{keV}$ (Hayashida 1990; Warwick 1991). The Ginga detection limit is determined by the source confusion rather than counting statistics. This source-confusion noise causes the sky brightness flucuation. Hence, the observed sky brightness distribution allows us to extend the $\log \mathrm{N}-\log \mathrm{S}$ relation to the S-values well below the source detection linit, as shown in Fig. 2. Thus obtained $\log \mathrm{N}-\operatorname{logS}$ relation shows that the source distribution is consistent to be Euclidean down to $S \sim 10^{-13} \mathrm{ergs} / \mathrm{cm}^{2} \mathrm{~S}$ in the $2-10 \mathrm{keV}$ band, and that sources brighter than $10^{-13} \mathrm{ergs} / \mathrm{cm}^{2} \mathrm{~s}$ account for as much as $30 \%$ of $\mathrm{CXB}$ of this energy range.

The average energy spectrum of the sources responsible for the observed sky brightness fluctuation is also determined. As a result, the average energy index in the $2-10 \mathrm{keV}$ range for those sources mostly with fluxes a little below $10^{-12} \mathrm{ergs} / \mathrm{cm}^{2} \mathrm{~s}$ is $0.67(0.64-0.72$; the $90 \%$ confidence limits) (Hayashida 1990). This suggests that CXB down to this flux range is still dominated by those AGNs having seyfert 1 type spectra.

On the other hand, the spectrum of CXB is quite different: an index of 0.4 in the $2-20 \mathrm{keV}$ range with a distinct steepening to 1.6 in higher energies such as characterized by a bremsstrahlung spectrum of kT $\sim 40 \mathrm{keV}$ (Marshall et al. 19980). There is not much room left for accomodating many more seyfert 1 type AGNs than the Ginga result, unless their spectra are significantly steepened above $20 \mathrm{keV}$. Thus, most of the fainter unresolved sources below the Ginga limit $\left(<10^{-12} \mathrm{ergs} / \mathrm{cm}^{2} \mathrm{~s}\right)$ must have unique spectra with an average index smaller than 0.4 below 20 $\mathrm{keV}$ and a more pronounced high-energy break than that of the CXB. 


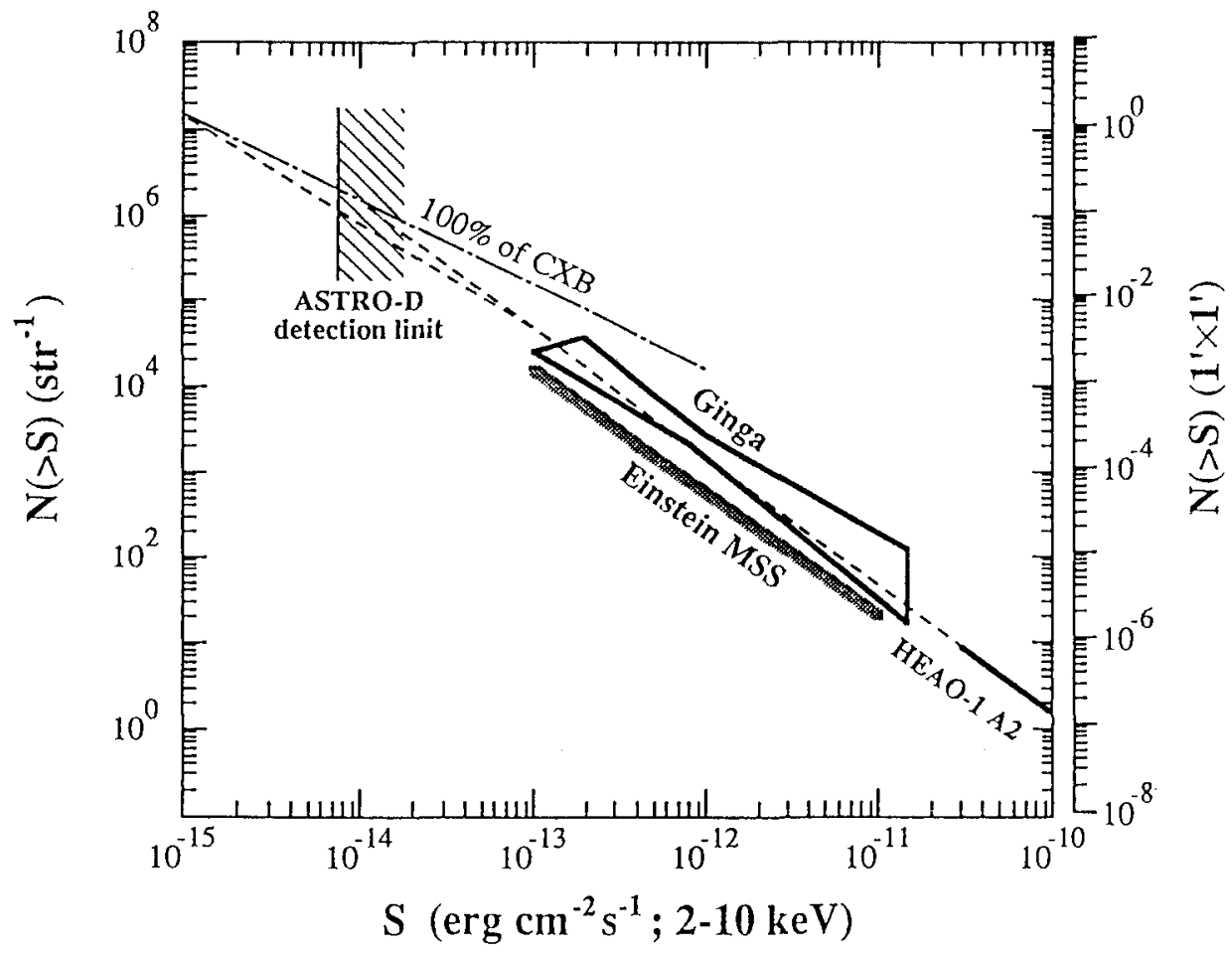

Fig. 2. $\log \mathrm{N}-\log \mathrm{S}$ relation derived from sky brightness fluctuation.

Recently, attemps have been made to resolve the "spectral paradox" by invoking a dominant contribution of the Compton-reflected component of AGNs (see Section 2.) (e.g. Fabian 1990; Terasawa 1991). Compton reflection is expected to cause a spectral steepening which is characterized approximately by that of a thermal bremsstrahlung spectrum of $\mathrm{kT} \sim 120 \mathrm{keV}$. In order to reproduce a fairly sharp break of the CXB spectrum this way, a dominant contribution must come from a relatively narrow range of $\mathrm{z}$ around 3 . In any event, it is an inevitable conclusion that a large change in spectral shape should occur as the source flux goes below $10^{-13} \mathrm{ergs} / \mathrm{cm}^{2} \mathrm{~s}$.

Next high-energy $X$-ray observations including Astro-D will not only resolve fainter sources but are also expected to find a rapid spectral evolution, or eventually a different class of objects.

\section{REFERENCES.}

Awaki,H. et al. (1990), Nature, 346, 544.

Awaki,H. (1991), ISAS Research Note, RN 473.

Cavallo,G. and Rees,M.J. (1978), M.N.R.A.S.,183, 359. 
Fabian,A.C. (1979), Proc. Royal Soc. London, A, 366, 449.

Fabian,A.C. et al. (1990), M.N.R.A.S., 242, 14.

Ilayashida,K. (1990), ISAS Research Note, RN 466.

Makino,F. (1989), Astrophys. J. Letters, 347, L9.

Matsuoka, M. et al. (1990), Astrophys. J., 361, 440 .

Marshall,F.E. et al. (1980), Astrophys. J., 235, 4.

Pounds,K.A. et al. (1990), Nature, 344, 132.

Remillard,R.A. et al. (1991), Nature, 350, 589.

Tanaka,Y., (1990), Proc. Workshop on "Iron Line Diagnostics in X-Ray Sources", Varenna, 1990.

Tanaka,Y., Proceedings of this Joint Discussion.

Terasawa,N. (1991), Astrophys. J. Letters, 378, L11.

Warwick,R.S. and Stewart,G.C. (1989), Proc. 23rd ESLAB Symp., Bologna, 1989,2, p.727. 